\title{
ON THE $\alpha$-CLASSIFICATION OF BIRTH-DEATH AND QUASI-BIRTH-DEATH PROCESSES
}

Erik A. van Doorn $\square \quad$ Department of Applied Mathematics, University of Twente, The Netherlands

\begin{abstract}
$\square \quad$ In several recent papers criteria for the $\alpha$-classification of birth-death and quasi-birth-death processes have been proposed. In this paper the relations between the various criteria are brought to light.
\end{abstract}

Keywords Associated polynomials; Decay parameter; Karlin-McGregor representation; Orthogonal polynomials; Spectral measure.

2000 Mathematics Subject Classification Primary 60J80.

\section{INTRODUCTION}

The $\alpha$-classification of birth-death processes has recently been studied in papers by Hart, Martínez, and San Martín ${ }^{[10]}$ and the present author ${ }^{[9]}$. The purpose of this paper is to display the relation between the results in both papers. Moreover, the correspondence between the classification results for birth-death processes and those for quasi-birth-death processes obtained (for discrete-time processes) by Bean, Pollett, and Taylor ${ }^{[2]}$ will be brought to light.

\section{PRELIMINARIES}

We consider a birth-death process $\mathscr{X}:=\{X(t), t \geq 0\}$ taking values in $S:=\{0,1, \ldots\}$ with birth rates $\left\{\lambda_{n}, n \in S\right\}$ and death rates $\left\{\mu_{n}, n \in S\right\}$, all strictly positive. Positivity of $\mu_{0}$ entails that the process may evanesce by escaping from $S$, via state 0 , to an absorbing state -1 . 
Karlin and McGregor ${ }^{[11]}$ have shown that the transition probabilities

$$
p_{i j}(t):=\operatorname{Pr}\{X(t)=j \mid X(0)=i\}, \quad t \geq 0, \quad i, j \in S,
$$

can be represented as

$$
p_{i j}(t)=\pi_{j} \int_{0}^{\infty} e^{-x t} Q_{i}(x) Q_{j}(x) \psi(d x), \quad t \geq 0, \quad i, j \in S .
$$

Here, $\left\{\pi_{n}\right\}$ are constants given by

$$
\pi_{0}:=1 \quad \text { and } \quad \pi_{n}:=\frac{\lambda_{0} \lambda_{1} \ldots \lambda_{n-1}}{\mu_{1} \mu_{2} \ldots \mu_{n}}, \quad n>0
$$

$\left\{Q_{n}(x)\right\}$ is a sequence of polynomials satisfying the recurrence relation

$$
\begin{aligned}
\lambda_{n} Q_{n+1}(x) & =\left(\lambda_{n}+\mu_{n}-x\right) Q_{n}(x)-\mu_{n} Q_{n-1}(x), \quad n \geq 1, \\
\lambda_{0} Q_{1}(x) & =\lambda_{0}+\mu_{0}-x, \quad Q_{0}(x)=1,
\end{aligned}
$$

and $\psi-$ the spectral measure of $\mathscr{X}$ - is a Borel measure of total mass 1 on the interval $(0, \infty)$ with respect to which the birth-death polynomials $\left\{Q_{n}(x)\right\}$ are orthogonal. We let, for $s \in \mathbb{R}$ sufficiently small,

$$
\tilde{p}_{i j}(s):=\int_{0}^{\infty} e^{s t} p_{i j}(t) d t \quad i, j \in S,
$$

and note for future reference that upon the substitution of representation (1) in (3) and the interchange of integrals, we obtain, for $i=j=0$,

$$
\tilde{p}_{00}(s)=\int_{0}^{\infty} \frac{\psi(d x)}{x-s}
$$

the Stieltjes transform of the measure $\psi$.

In what follows (as in Refs..$^{[9,10]}$ ) we shall assume that the spectral measure of $\mathscr{X}$, and hence the process $\mathscr{X}$ itself, is uniquely determined by its birth and death rates. An important role will be played by the quantities $\xi_{i}$, recurrently defined by

$$
\xi_{1}:=\inf \operatorname{supp}(\psi)
$$

where $\operatorname{supp}(\psi)$ denotes the support of $\psi$, and

$$
\xi_{i+1}:=\inf \left\{\operatorname{supp}(\psi) \cap\left(\xi_{i}, \infty\right)\right\}, \quad i \geq 1
$$


We also let

$$
\sigma:=\lim _{i \rightarrow \infty} \xi_{i}
$$

the first accumulation point of $\operatorname{supp}(\psi)$ if it exists, and infinity otherwise. Under our assumption that $\psi$ is uniquely determined by the birth and death rates of the process, the quantities $\xi_{i}$ and $\sigma$ may be defined alternatively in terms of the (simple and positive) zeros of the polynomials $Q_{n}(x)$ (see Chihara ${ }^{[4]}$ Section II.4). Namely, with $x_{n 1}<x_{n 2}<\cdots<x_{n n}$ denoting the $n$ zeros of $Q_{n}(x)$, we have the classic separation result

$$
0<x_{n+1, i}<x_{n i}<x_{n+1, i+1}, \quad i=1,2, \ldots, n, \quad n \geq 1,
$$

so that the limits as $n \rightarrow \infty$ of $x_{n i}$ exist, and

$$
\lim _{n \rightarrow \infty} x_{n i}=\xi_{i}, \quad i=1,2, \ldots, n .
$$

Given the birth-death polynomials $\left\{Q_{n}(x)\right\}$ one defines the corresponding sequence of associated polynomials $\left\{Q_{n}^{(k)}(x)\right\}$ of order $k, k \geq 1$, by replacing $\lambda_{n}$ and $\mu_{n}$ by $\lambda_{n+k}$ and $\mu_{n+k}$, respectively, in the recurrence relation (2), so that the polynomials $\left\{Q_{n}^{(k)}(x)\right\}$ satisfy the recurrence

$$
\begin{aligned}
\lambda_{n+k} Q_{n+1}^{(k)}(x) & =\left(\lambda_{n+k}+\mu_{n+k}-x\right) Q_{n}^{(k)}(x)-\mu_{n+k} Q_{n-1}^{(k)}(x), \quad n \geq 1, \\
\lambda_{k} Q_{l}^{(k)}(x) & =\lambda_{k}+\mu_{k}-x, \quad Q_{0}^{(k)}(x)=1 .
\end{aligned}
$$

The associated polynomials of order $k$ are orthogonal with respect to a (unique) measure $\psi^{(k)}$ (the associated measure of order $k$ ), which is also the spectral measure of the birth-death process $\mathscr{X}^{(k)}$ (the associated process of order $k$ ) with birth rates $\left\{\lambda_{n}^{(k)}:=\lambda_{n+k}, n \in S\right\}$ and death rates $\left\{\mu_{n}^{(k)}:=\mu_{n+k}, n \in S\right\}$. It will be convenient to allow $k=0$ in the above definitions; the resulting quantities should be identified with those without superscripts. For example, $\mathscr{X}^{(0)}=\mathscr{X}$.

A classic result in the theory of continued fractions (see, for example, Berg $\left.^{[3]}\right)$ tells us that the Stieltjes transforms of the spectral measures $\psi^{(k)}$ and $\psi^{(k+1)}$ are related as

$$
\int_{0}^{\infty} \frac{\psi^{(k)}(d x)}{x-s}=\left(\lambda_{k}+\mu_{k}-s-\lambda_{k} \mu_{k+1} \int_{0}^{\infty} \frac{\psi^{(k+1)}(d x)}{x-s}\right)^{-1}, \quad k \geq 0 .
$$

So, in view of (4) and in an obvious notation, we have

$$
\tilde{p}_{00}^{(k)}(s)=\left(\lambda_{k}+\mu_{k}-s-\lambda_{k} \mu_{k+1} \tilde{p}_{00}^{(k+1)}(s)\right)^{-1}, \quad k \geq 0,
$$

a result that will prove useful in Section 5 . 
Defining $\xi_{i}^{(k)}$ and $\sigma^{(k)}$ as in $(5)-(7)$ with $\psi$ replaced by $\psi^{(k)}$, we have (see Ref. ${ }^{[5]}$, Section II.4 and Theorem III.4.2)

$$
0 \leq \xi_{i}^{(k)} \leq \xi_{i}^{(k+1)} \leq \xi_{i+1}^{(k)}<\infty, \quad k \geq 0, \quad i \geq 1
$$

whence

$$
\sigma^{(k+1)}=\sigma^{(k)}=\sigma, \quad k \geq 0
$$

while it is clear from the definition of $\xi_{i}^{(k)}$ that, for all $k \geq 0$ and $i \geq 1$,

$$
\xi_{i}^{(k)}=\xi_{i+1}^{(k)} \Longleftrightarrow \xi_{i}^{(k)}=\sigma .
$$

We also have (see Ref. ${ }^{[5]}$, Theorems IV.2.1 and IV.3.2 or Ref. ${ }^{[6]}$, Theorem 1)

$$
\lim _{k \rightarrow \infty} \xi_{i}^{(k)}=\sigma, \quad i \geq 1
$$

while it follows from Theorem 3 Chihara $^{[4]}$ (see also Ref. ${ }^{[8]}$, Corollary 3.2) that, for all $k \geq 0$ and $i \geq 1$,

$$
\xi_{i}^{(k)}=\xi_{i}^{(k+1)} \Longleftrightarrow \xi_{i}^{(k)}=\sigma .
$$

From the general theory of continuous-time Markov chains (see, for example, Anderson ${ }^{[1]}$ ) we know that there exists a number $\alpha \geq 0$, called the decay parameter of $\mathscr{X}$, such that for each pair $i, j \in S$

$$
\lim _{t \rightarrow \infty} \frac{1}{t} \log p_{i j}(t)=-\alpha .
$$

The decay parameter $\alpha$ may be interpreted as the common abscissa of convergence of the Laplace transforms $\tilde{p}_{i j}(s)$, that is,

$$
\alpha=\sup \left\{s \geq 0: \tilde{p}_{i j}(s)<\infty\right\}, \quad i, j \in S .
$$

(Note that $\tilde{p}_{i j}(0)<\infty$ since $\mathscr{X}$ is transient.) Representation formula (1) is easily seen to imply (see, for example, Ref. ${ }^{[7]}$ ) that $\alpha$ is also the smallest point in the support of the spectral measure $\psi$, that is,

$$
\alpha=\xi_{1}
$$

The process $\mathscr{X}$ is said to be $\alpha$-recurrent if for some state $i \in S$ (and then for all states $i \in S$ )

$$
\tilde{p}_{i i}(\alpha)=\int_{0}^{\infty} e^{\alpha t} p_{i i}(t) d t=\infty
$$


and $\alpha$-transient otherwise. An $\alpha$-recurrent process is said to be $\alpha$-positive if for some state $i \in S$ (and then for all states $i \in S$ )

$$
\lim _{t \rightarrow \infty} e^{\alpha t} p_{i i}(t)>0
$$

and $\alpha$-null otherwise. The $\alpha$-classification of the process $\mathscr{X}$ amounts to establishing whether $\mathscr{X}$ is $\alpha$-transient, $\alpha$-null, or $\alpha$-positive. Since we have assumed $\mu_{0}>0$, the birth-death process $\mathscr{X}$ is transient, and hence $\alpha$ transient if $\alpha=0$.

\section{3. $\alpha$-CLASSIFICATION OF BIRTH-DEATH PROCESSES}

The following theorem has been proven in Ref. ${ }^{[9]}$, where, however, $\mu_{0}$ in condition (iv) below was inadvertently omitted in the statement of the theorem. Here and in what follows we use the convention that for any real $c$

$$
\int_{c}^{\infty} \frac{\psi^{(k)}(d x)}{x-c}:=\lim _{s \uparrow c} \int_{c}^{\infty} \frac{\psi^{(k)}(d x)}{x-s}, \quad k \geq 0 .
$$

Theorem 3.1 (Ref. $^{[9]}$ Theorem 5.2). Let the birth-death process $\mathscr{X}$ have decay parameter $\alpha$. Then the following are equivalent:

(i) $\mathscr{X}$ is $\alpha$-recurrent,

(ii) $\sum_{n=0}^{\infty}\left(\lambda_{n} \pi_{n} Q_{n}(\alpha) Q_{n+1}(\alpha)\right)^{-1}=\infty$,

(iii) $\int_{\alpha}^{\infty} \frac{\psi(d x)}{x-\alpha}=\infty$,

(iv) $\int_{\alpha}^{\infty} \frac{\psi^{(1)}(d x)}{x-\alpha}=\frac{\lambda_{0}+\mu_{0}-\alpha}{\lambda_{0} \mu_{1}}$;

moreover, if $\mathscr{X}$ is $\alpha$-recurrent then the following are equivalent:

(v) $\mathscr{X}$ is $\alpha$-positive;

(vi) $\sum_{n=0}^{\infty} \pi_{n} Q_{n}^{2}(\alpha)<\infty$,

(vii) $\psi(\{\alpha\})>0$,

(viii) $\int_{\alpha}^{\infty} \frac{\psi^{(1)}(d x)}{(x-\alpha)^{2}}<\infty$.

We note that, by Theorem 2.2 Ref. $^{[9]}$ and the argument leading to Theorem 3.1, we always have

$$
\int_{\alpha}^{\infty} \frac{\psi^{(1)}(d x)}{x-\alpha} \leq \frac{\lambda_{0}+\mu_{0}-\alpha}{\lambda_{0} \mu_{1}}<\infty
$$

so that $\mathscr{X}$ is $\alpha$-transient if and only if the first inequality in (20) is strict.

The main findings of Ref. ${ }^{[10]}$ may be stated as a corollary to Theorem 3.1 as follows, where $\alpha_{\ell}$ denotes the decay parameter of $\mathscr{X}^{(\ell)}$. 
Corollary 3.1 (Ref. ${ }^{[10]}$ Theorem 1 and Corollary). Let $k \geq 0$.

(i) If $\xi_{1}^{(k)}<\xi_{1}^{(k+1)}$ then $\mathscr{X}^{(k)}$ is $\alpha_{k}$-positive,

(ii) if $\xi_{1}^{(k)}=\xi_{1}^{(k+1)}$ then $\mathscr{X}^{(\ell)}$ is $\alpha_{\ell}$-transient for $\ell \geq k+1$.

Proof. (i) If $\alpha_{k}=\xi_{1}^{(k)}<\xi_{1}^{(k+1)}$ then, since $\xi_{1}^{(k+1)} \leq \sigma^{(k+1)}=\sigma^{(k)}$, the smallest accumulation point of $\operatorname{supp}\left(\psi^{(k)}\right), \psi^{(k)}$ must have a point mass at $\xi_{1}^{(k)}$. It follows that conditions (iii) and (vii) of Theorem 3.1 are satisfied, so that $\mathscr{L}^{(k)}$ is $\alpha_{k}$-positive.

(ii) If $\xi_{1}^{(k)}=\xi_{1}^{(k+1)}$ then, by (16) and (17), $\alpha_{k}=\xi_{1}^{(k)}=\sigma=\xi_{1}^{(\ell)}=\alpha_{\ell}$ for all $\ell \geq k+1$. Hence, interpreting (20) in terms of $\mathscr{X}^{(\ell-1)}$ rather than $\mathscr{X}$, we find

$$
\int_{\alpha_{\ell}}^{\infty} \frac{\psi^{(\ell)}(d x)}{x-\alpha_{\ell}}=\int_{\alpha_{\ell-1}}^{\infty} \frac{\psi^{(\ell)}(d x)}{x-\alpha_{\ell-1}}<\infty, \quad \ell \geq k+1
$$

It follows that for all $\ell \geq k+1$ the analogue of condition (iii) of Theorem 3.1 for $\mathscr{X}^{(\ell)}$ is not satisfied, so that $\mathscr{L}^{(\ell)}$ is $\alpha_{\ell}$-transient.

Choosing $k=0$ for convenience, and considering that, by (17), $\xi_{1}$ is an isolated point in $\operatorname{supp}(\psi)$ if and only if $\xi_{1}<\xi_{1}^{(1)}$, we may reformulate the preceding corollary by stating that $\mathscr{X}$ is $\alpha$-positive if the smallest point of $\operatorname{supp}(\psi)$ is isolated, while $\mathscr{L}^{(\ell)}$ is $\alpha_{\ell}$-transient for all $\ell \geq 1$ otherwise.

The corollary does not say anything about the $\alpha$-classification of $\mathscr{X}$ when $\alpha=\sigma$, so in Ref. ${ }^{[10]}$ the authors pose this as an open problem, and suggest that a situation analogous to that described by Bean, Pollett, and Taylor $^{[2]}$ for discrete-time quasi-birth-death processes might prevail. This will be confirmed in Section 5. The open problem itself is resolved by Theorem 3.1, which, actually, enables us to perform the $\alpha$-classification of $\mathscr{X}$ if we know the spectral measure $\psi$ of $\mathscr{X}$ in a small neighbourhood of $\alpha$ (or the measure $\psi^{(1)}$ of the associated process $\mathscr{X}^{(1)}$ ).

It is enlightening to realize that any probability measure on $[0, \infty)$ with finite moments of all orders can be interpreted as the spectral measure of a birth-death process. Indeed, any such measure defines a positive-definite moment functional and hence a sequence of orthogonal polynomials, which (see the Corollary to Theorem I.9.1 in Ref. ${ }^{[5]}$ ) can be identified as a sequence of birth-death polynomials. If the measure has a finite moment of order -1 then one can choose $\mu_{0}>0$. With these facts in mind it is obvious that there exist (absorbing) birth-death processes that have $\alpha=\sigma$, and are $\alpha$-transient, $\alpha$-null, or $\alpha$-positive, respectively. Such processes have been constructed explicitly (in terms of birth and death rates) in Ref. ${ }^{[10]}$ (Section 5), but to prove their existence it suffices to note the existence of probability measures with finite moments of all orders, $\xi_{1}=\sigma$, and the 
appropriate properties imposed by Theorem 3.1. It follows in particular that the reverse implication in Corollary 3.1(i) does not hold.

We note that Corollary 3.1 does not say anything either about the $\alpha_{\ell^{-}}$ classification of $\mathscr{X}^{(\ell)}$ for $\ell \geq 1$ when $\alpha=\xi_{1}<\sigma$. But again, anything can happen. Indeed, if $\psi$ is such that $\xi_{1}<\xi_{2}<\sigma$ then $\alpha_{1}=\xi_{1}^{(1)}<\sigma$, and hence $\mathscr{X}^{(1)}$ is $\alpha_{1}$-positive. If, on the other hand, $\psi$ is such that $\xi_{1}<\xi_{2}=\sigma$ then, using the technique employed in Ref. ${ }^{[8]}$ (Section 3) and letting

$$
A:=\frac{\psi\left(\left\{\xi_{1}\right\}\right)}{\xi_{1}-\sigma}+\int_{\sigma}^{\infty} \frac{\psi(d x)}{x-\sigma},
$$

we find again that $\xi_{1}^{(1)}<\sigma$ (and hence $\mathscr{X}^{(1)}$ is $\alpha_{1}$-positive) if $A<0$, but $\xi_{1}^{(1)}=\sigma$ if $A \geq 0$. Further analysing the latter case in a similar fashion, we find that

$$
\int_{\alpha_{1}}^{\infty} \frac{\psi^{(1)}(d x)}{x-\alpha_{1}}=\int_{\sigma}^{\infty} \frac{\psi^{(1)}(d x)}{x-\sigma}<\infty
$$

- and hence (by Theorem 3.1) $\mathscr{X}^{(1)}$ is $\alpha_{1}$-transient - if $A>0$, whereas the integral in (21) diverges - and hence $\mathscr{X}^{(1)}$ is $\alpha_{1}$-recurrent - if $A=0$. In the latter case $\mathscr{X}^{(1)}$ may be $\alpha_{1}$-null as well as $\alpha_{1}$-positive (examples of both can be given). We note in particular that the reverse implication in Corollary 3.1(ii) does not hold.

\section{QUASI-BIRTH-DEATH PROCESSES}

We now widen our perspective by allowing $\mathscr{X}:=\{X(t), t \geq 0\}$ to be a (level-dependent) quasi-birth-death process. Hence $\mathscr{X}$ has a twodimensional state space $S:=\left\{(k, j) \mid k \geq 0, j=1,2, \ldots, J_{k}\right\}$, with the first dimension corresponding to subsets of states called levels, and the second (finite) dimension corresponding to the phase in each level. Moreover, the $q$-matrix $Q$ of $\mathscr{X}$ takes the block-partitioned form

$$
Q:=\left(\begin{array}{cccccccc}
N_{0} & \Lambda_{0} & 0 & 0 & 0 & . & . & . \\
M_{1} & N_{1} & \Lambda_{1} & 0 & 0 & . & . & . \\
0 & M_{2} & N_{2} & \Lambda_{2} & 0 & . & . & . \\
. & . & . & . & . & . & . & . \\
. & . & . & . & . & . & . & .
\end{array}\right)
$$

where $\Lambda_{k}, N_{k}, k \geq 0$, and $M_{k}, k \geq 1$, are nonzero matrices of order $J_{k} \times$ $J_{k+1}, J_{k} \times J_{k}$, and $J_{k} \times J_{k-1}$, respectively. In what follows we assume $\mathscr{X}$ to be uniquely determined by $Q$. We shall also assume that $S$ is irreducible, and

$$
N_{0} \mathbf{1}+\Lambda_{0} \mathbf{1}<\mathbf{0}
$$


where $\mathbf{0}$ and $\mathbf{1}$ are column vectors of zeros and ones, respectively, of appropriate length, and strict inequality for vectors indicates strict inequality in at least one component. Hence, whatever the initial state, the process may escape from $S$, via at least one state at level 0 , to an (ignored) absorbing state -1 . Evidently, if $J_{k}=1$ for all levels $k$ then we are back in the setting of simple birth-death processes.

Generalizing some concepts of the previous sections we define the associated process of order $k$ to be the process $\mathscr{X}^{(k)}:=\left\{X^{(k)}(t), t \geq 0\right\}$ with state space $S$ and $q$-matrix

$$
Q^{(k)}:=\left(\begin{array}{cccccccc}
N_{k} & \Lambda_{k} & 0 & 0 & 0 & . & . & . \\
M_{k+1} & N_{k+1} & \Lambda_{k+1} & 0 & 0 & . & . & . \\
0 & M_{k+2} & N_{k+2} & \Lambda_{k+2} & 0 & . & . & . \\
\cdot & \cdot & \cdot & . & . & \cdot & . & . \\
\cdot & \cdot & . & . & . & . & . & .
\end{array}\right), \quad k \geq 0
$$

so that $\mathscr{X}^{(0)}=\mathscr{X}$. In what follows $\mathscr{X}^{(k)}$ will actually be assumed to be irreducible for all $k \geq 0$, a property called total irreducibility in Ref. ${ }^{[2]}$. Since the $M_{k}$ are nonzero matrices it follows that the processes $\mathscr{L}^{(k)}$ are absorbing (and hence transient) for all $k \geq 0$.

We let

$$
p_{i j}^{(k)}(t):=\operatorname{Pr}\left\{X^{(k)}(t)=j \mid X^{(k)}(0)=i\right\}, \quad i, j \in S, \quad k \geq 0, \quad t \geq 0 .
$$

and denote the decay parameter of $\mathscr{X}^{(k)}$ in $S$ by $\alpha_{k}$. Hence,

$$
\alpha_{k}=\sup \left\{s \geq 0: \tilde{p}_{i j}^{(k)}(s)<\infty\right\}, \quad i, j \in S, k \geq 0,
$$

where

$$
\tilde{p}_{i j}^{(k)}(s):=\int_{0}^{\infty} e^{s t} p_{i j}^{(k)}(t) d t, \quad i, j \in S, \quad k \geq 0 .
$$

(Note that $\tilde{p}_{i j}^{(k)}(0)<\infty$ since $\mathscr{X}^{(k)}$ is transient.) The process $\mathscr{L}^{(k)}$ may now be classified as $\alpha_{k}$-recurrent or $\alpha_{k}$-transient, depending on whether $\tilde{p}_{i i}^{(k)}\left(\alpha_{k}\right)$ is infinite or finite, respectively, for some (and then each) state $i \in S$, while an $\alpha_{k}$-recurrent process is $\alpha_{k}$-positive or $\alpha_{k}$-null depending on whether

$$
\lim _{t \rightarrow \infty} e^{\alpha_{k} t} p_{i i}^{(k)}(t)
$$

is positive or zero, respectively, for some (and then each) state $i \in S$. Criteria for the $\alpha_{k}$-classification of $\mathscr{X}^{(k)}$ have been obtained in Ref. ${ }^{[2]}$ in a discrete-time setting. In the next section we will briefly describe the continuous-time analogues of these results, and we will show that the 
classification results reduce to results given in Section 3 when $J_{k}=1$ for all $k$. The proofs of the statements about continuous-time quasi-birth-death processes are straightforward analogues of their discrete-time counterparts in Ref. ${ }^{[2]}$, and will not be given explicitly.

\section{5. $\alpha$-CLASSIFICATION OF QUASI-BIRTH-DEATH PROCESSES}

We need some further notation. For all $k \geq 0$ we let $T^{(k)}$ be the (defective) random variable representing the first entrance time into level 0 of the process $\mathscr{X}^{(k)}$, that is, the time it takes for a transition into a state at level 0 to occur for the first time. We also let, for $i, j=1,2, \ldots, J_{k}$, and $k \geq 0$,

$$
F_{i j}^{(k)}(t):=\operatorname{Pr}\left\{T^{(k)} \leq t, X^{(k)}\left(T^{(k)}+\right)=(0, j) \mid X^{(k)}(0)=(0, i)\right\}, \quad t \geq 0,
$$

and

$$
\tilde{f}_{i j}^{(k)}(s):=\int_{0}^{\infty} e^{s t} d F_{i j}^{(k)}(t) .
$$

By $\widetilde{P}_{k}(s)$ and $\widetilde{F}_{k}(s)$ we denote the $J_{k} \times J_{k}$ matrices with elements $\tilde{p}_{(0, i),(0, j)}^{(k)}(s)$ and $\tilde{f}_{i j}^{(k)}(s)$, respectively, and $\Phi_{k}(s)$ is the $J_{k} \times J_{k}$ matrix

$$
\Phi_{k}(s):=-\operatorname{diag}\left(s+\left[N_{k}\right]_{11}, s+\left[N_{k}\right]_{22}, \ldots, s+\left[N_{k}\right]_{J_{k}, J_{k}}\right), \quad k \geq 0 .
$$

After some reflection it becomes clear that the relations between $\widetilde{P}_{k}(s)$ and $\widetilde{F}_{k}(s)$ - the continuous-time analogues of Ref. ${ }^{[2]}((3.3)-(3.5))$ - are given by

$$
\widetilde{F}_{k}(s)=\Phi_{k}^{-1}(s)\left(I+\Phi_{k}^{-1}(0) N_{k}+\Lambda_{k} \widetilde{P}_{k+1}(s) M_{k+1}\right), \quad k \geq 0,
$$

where $I$ is the $J_{k} \times J_{k}$ identity matrix, and

$$
\widetilde{P}_{k}(s)=\Phi_{k}^{-1}(s)+\sum_{n=1}^{\infty}\left(\widetilde{F}_{k}(s)\right)^{n}, \quad k \geq 0 .
$$

Total irreducibility is readily seen to imply that $\alpha_{k} \leq \alpha_{k+1}$ for all $k$, while it is well known that, for all $k \geq 0$,

$$
\alpha_{k} \leq \inf _{\ell \geq k} \inf _{i}\left\{-\left[N_{\ell}\right]_{i i}\right\}
$$

(see, for example, Ref. ${ }^{[1]}$ Theorem 5.1.9). So we see from (25) and (26) that, for $k \geq 0$,

$$
s<\alpha_{k} \Longrightarrow \tilde{f}_{i j}^{(k)}(s)<\infty .
$$


Proceeding as in Ref. ${ }^{[2]}$, we let $\eta_{k}(s)$ be the Perron-Frobenius eigenvalue of $\widetilde{F}_{k}(s)$, which is a well-defined, continuous and strictly increasing function for $s<\alpha_{k}$. Moreover, using (27) it can easily be shown that

$$
\eta_{k}\left(\alpha_{k}\right):=\lim _{s \uparrow \alpha_{k}} \eta_{k}(s) \leq 1, \quad k \geq 0
$$

The analogues in continuous time of the $\alpha$-classification results for discretetime quasi-birth-death processes in Ref. ${ }^{[2]}$ (Lemma 15) may now be summarized as follows, where, for convenience, we take $k=0$ and write $\eta:=\eta_{0}$.

Theorem 5.1. Let the quasi-birth-death process $\mathscr{X}$ have decay parameter $\alpha$. Then $\mathscr{X}$ is $\alpha$-recurrent if and only if $\eta(\alpha)=1$; the process $\mathscr{X}$ is $\alpha$-positive if, in addition,

$$
\left[\frac{d}{d s} \tilde{p}_{(0, i),(0, j)}^{(1)}(s)\right]_{s=\alpha}<\infty, \quad i, j=1,2, \ldots, J_{0},
$$

and $\alpha$-null otherwise.

If $J_{k}=1$ for all $k \geq 0$, then the quasi-birth-death process $\mathscr{X}^{(k)}$ of the previous section is an ordinary birth-death process with birth rates $\lambda_{k}=\Lambda_{k}$ and death rates $\mu_{k}=M_{k}$. Hence, the matrix $\widetilde{F}_{k}(s)$ is in fact a scalar, and its Perron-Frobenius eigenvalue $\eta_{k}(s)$ is simply $\widetilde{F}_{k}(s)$ itself. Moreover, (26) tells us that

$$
\eta_{k}(s)=\widetilde{F}_{k}(s)=\frac{\lambda_{k} \mu_{k+1} \widetilde{P}^{(k+1)}(s)}{\lambda_{k}+\mu_{k}-s}, \quad k \geq 0 .
$$

But from (12) we obtain

$$
\lambda_{k} \mu_{k+1} \widetilde{P}^{(k+1)}(s)=\lambda_{k} \mu_{k+1} \tilde{p}_{00}^{(k+1)}(s)=\lambda_{k}+\mu_{k}-s-\left(\tilde{p}_{00}^{(k)}(s)\right)^{-1},
$$

so that we can actually write

$$
\eta_{k}(s)=1-\left(\left(\lambda_{k}+\mu_{k}-s\right) \tilde{p}_{00}^{(k)}(s)\right)^{-1}, \quad k \geq 0 .
$$

Substitution of the representation (4) (in terms of $\mathscr{X}^{(k)}$ ) in (32), and considering that, in view of (8) and (9) (in terms of $\mathscr{L}^{(k)}$ again),

$$
\alpha_{k}=\xi_{1}^{(k)}<x_{11}^{(k)}=\lambda_{k}+\mu_{k}
$$

we can take the limit $s \uparrow \alpha_{k}$ in (32), and conclude that, for all $k \geq 0$,

$$
\eta_{k}\left(\alpha_{k}\right)=1 \Longleftrightarrow \int_{\alpha_{k}}^{\infty} \frac{\psi^{(k)}(d x)}{x-\alpha_{k}}=\infty .
$$


Moreover, differentiating (4) (in terms of $\mathscr{X}^{(k+1)}$ ) and taking the limit $s \uparrow \alpha_{k}$ again, gives us, for all $k \geq 0$,

$$
\left[\frac{d}{d s} \tilde{p}_{00}^{(k+1)}(s)\right]_{s=\alpha_{k}}<\infty \Longleftrightarrow \int_{\alpha_{k}}^{\infty} \frac{\psi^{(k+1)}(d x)}{\left(x-\alpha_{k}\right)^{2}}<\infty .
$$

Choosing $k=0$ in (33) and (34) shows that when $J_{k}=1$ for all $k$, the results of Theorem 5.1 are implied by Theorem 3.1, as announced.

\section{ACKNOWLEDGEMENT}

The author wishes to thank Nigel Bean and Peter Taylor for sharing their thoughts on the interpretation for simple birth-death processes of the results in Ref. ${ }^{[2]}$, which has triggered the analysis in Section 5.

\section{REFERENCES}

1. Anderson, W.J. Continuous-time Markov Chains; Springer: New York, 1991.

2. Bean, N.G.; Pollett, P.K.; Taylor, P.G. The quasistationary distributions of level-dependent quasibirth-and-death processes. Stoch. Models 2000, 16, 511-541.

3. Berg, C. Markov's theorem revisited. J. Approx. Theory 1994, 78, 260-275.

4. Chihara, T.S. On co-recursive orthogonal polynomials. Proc. Amer. Math. Soc. 1957, 8, 899-905.

5. Chihara, T.S. An Introduction to Orthogonal Polynomials; Gordon and Breach: New York, 1978.

6. van Doorn, E.A. On oscillation properties and the interval of orthogonality of orthogonal polynomials. SIAM J. Math. Anal. 1984, 15, 1031-1042.

7. van Doorn, E.A. Representations for the rate of convergence of birth-death processes. Theory Probab. Math. Statist. 2002, 65, 37-43.

8. van Doorn, E.A. On associated polynomials and decay rates for birth-death processes. J. Math. Anal. Appl. 2003, 278, 500-511.

9. van Doorn, E.A. Birth-death processes and associated polynomials. J. Comput. Appl. Math. 2003, 153, 497-506.

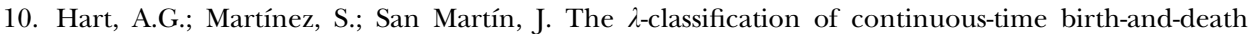
processes. Adv. Appl. Probab. 2003, 35, 1111-1130.

11. Karlin, S.; McGregor, J.L. The differential equations of birth-and-death processes, and the Stieltjes moment problem. Trans. Amer. Math. Soc. 1957, 85, 589-646. 